\title{
ASPP2א Is Expressed In Human Colorectal Carcinoma And Promotes Chemotherapy Resistance And Tumorigenesis
}

\section{OPEN ACCESS}

Edited by:

Kristoffer Vitting-Seerup, University of Copenhagen, Denmark

Reviewed by:

Guangrong Qin, Institute for Systems Biology (ISB), United States

Weiqi Rong,

Translational research, China

*Correspondence:

Kerstin M. Kampa-Schittenhelm Kerstin.kampa-schittenhelm@

kssg.ch

${ }^{\dagger}$ These authors have contributed equally to this work and share first authorship

¥These authors have contributed equally to this work and share last authorship

Specialty section:

This article was submitted to Protein and RNA Networks,

a section of the journal

Frontiers in Molecular Biosciences

Received: 18 June 2021

Accepted: 14 October 2021

Published: 05 November 2021

Citation:

Rieger I, Tsintari V, Overkamp M, Fend F, Lopez CD, Schittenhelm MM and Kampa-Schittenhelm KM (2021)

ASPP2 $\kappa$ Is Expressed In Human Colorectal Carcinoma And Promotes

Chemotherapy Resistance And Tumorigenesis.

Front. Mol. Biosci. 8:727203.

doi: 10.3389/fmolb.2021.727203
Ingmar Rieger ${ }^{1 \dagger}$, Vasileia Tsintari ${ }^{1 \dagger}$, Mathis Overkamp ${ }^{2}$, Falko Fend ${ }^{2}$, Charles D. Lopez ${ }^{3}$, Marcus M. Schittenhelm ${ }^{4 \neq}$ and Kerstin M. Kampa-Schittenhelm ${ }^{1,5 * \neq}$

${ }^{1}$ Department of Oncology, Hematology, Clinical Immunology and Rheumatology, University Hospital Tübingen (UKT), Tübingen, Germany, ${ }^{2}$ Institute of Pathology at the University Hospital Tübingen, Tübingen, Germany, ${ }^{3}$ Department of Hematology and Medical Oncology, Oregon Health and Science University (OHSU), Portland, OR, United States, ${ }^{4}$ Clinic of Medical Oncology and Hematology, Cantonal Hospital St. Gallen (KSSG), St. Gallen, Switzerland, ${ }^{5}$ Translational Experimental Hematology and Oncology, Medical Research Center and Department of Oncology and Hematology, Cantonal Hospital St. Gallen, St. Gallen, Switzerland

Alternative splicing is a common physiologic mechanism to generate numerous distinct gene products from one gene locus, which can result in unique gene products with differing important functional outcomes depending on cell context. Aberrant alternative splicing is a hallmark of cancer that can contribute to oncogenesis and aggressiveness of the disease as well as resistance to therapy. However, aberrant splicing might also result in novel targets for cancer therapy. ASPP2 is a haplo-insufficient tumor suppressor, that functions through both p53-dependent as well as p53independent mechanisms to enhance cell death after stress. Interestingly, the common human tumor TP53 mutations result in a loss of the binding sites to ASPP2, leading to impaired induction of apoptosis. Vice versa, attenuation of ASPP2 has been described to be associated with high-risk disease, therapy failure and poor clinical outcome especially in tumors harboring the TP53 wildtype (WT) isoform. We have recently identified a novel, dominant-negative splicing variant of ASPP2, named ASPP2 $\kappa$, with oncogenic potential. Exon-skipping results in a reading-frame shift with a premature translation stop, omitting most of the ASPP2 C-terminus - which harbors the p53-binding domain. Consequently, the ASPP2-p53 interaction is abrogated, which in part impacts on oncogenesis, aggressiveness of disease and response to therapy. Since ASPP $2 \kappa$ has been shown in hematologic malignancies to promote tumorigenesis, we further wished to determine if aberrant ASPP2 $\kappa$ expression plays a role in human solid tumors. In this report, we find that ASPP2 $k$ is frequently expressed in human colorectal tumors (CRC). Using ASPP2 $k$ overexpressing and interference CRC models, we demonstrate a functional role of ASPP2 $\kappa$ in contributing to oncogenesis and resistance to therapy in CRC by 1) enhancing proliferation, 2) promoting cell migration and, 3) confering resistance to chemotherapy induced apoptosis. Our findings have far-reaching consequences for future diagnostic and therapeutic strategies for ASPP2 $\kappa$ expressing colorectal cancer patients and provide proof-of-principle to further explore ASPP2 $\kappa$ as potential predictive marker and target for therapy in clinical trials.

Keywords: colon cancer, tumorigenesis, therapy resistance, alternative splicing, TP53, ASPP2, ASPP2к, apoptosis 


\section{INTRODUCTION}

Among all tumors, the incidence of colorectal cancer (CRC) ranks third worldwide in terms of incidence and second in terms of mortality (approx. 1.8 million, resp. 881,000 in total in 2018) (Bray et al., 2018). In the advanced metastatic stage, treatment options are mostly palliative except in selected circumstances (Chen et al., 2021). Moreover, despite increased knowledge and therapeutic options for metastatic CRC (Sveen et al., 2020), there remains a critical need for prognostic as well as predictive biomarkers to direct more effective therapies.

The current understanding of tumorigenesis supports the idea of a stepwise malignant transformation of cells by acquisition of alterations of genes that interfere with cell fate, cell survival, and genome maintenance (summarized by Vogelstein and colleagues (Vogelstein et al., 2013)). In CRC malignant transformation typically has a latency of several decades.

The tumor suppressor protein p53 (TP53), also referred to as "the guardian of the genome", plays a crucial role in the prevention of tumor formation and TP53 related apoptosis pathways are central mechanisms in cellular stress response and tumor suppression (Bergamaschi et al., 2004). Approximately 50\% of all colorectal cancers show TP53 inactivating gene mutations associated with poor clinical outcome (Yin et al., 2018). We and others have shown that dysregulation of the ASPP protein family displays an alternative mechanism to inactivate the p53 pathway, especially in TP53 wildtype (WT) cells (Vives et al., 2006; Kampa et al., 2009a). Two pro-apoptotic members (ASPP1 and ASPP2) and the anti-apoptotic variant (i)ASPP have been described (Bergamaschi et al., 2004; Trigiante and Lu, 2006; Sullivan and Lu, 2007). These proteins share an evolutionary conserved C-terminus including four-ankyrin repeats, a SH3-domain and a poly-proline-rich domain, which directly interact with the p53 core domain (Iwabuchi et al., 1994; Gorina and Pavletich, 1996; Samuels-Lev et al., 2001; Takahashi et al., 2004; Ahn et al., 2009).

ASPP1 and ASPP2 promote p53-dependent apoptosis (Iwabuchi et al., 1998). In contrast, iASPP binds to an adjacent linker region to inhibit apoptosis (Bergamaschi et al., 2006). Intriguingly, altered expression levels of ASPP1, ASPP2 or iASPP have been observed in CRC (Yin et al., 2018). However, the functional and clinical consequences of ASPP family isoforms have not been explored in CRC.

We now present data showing that the dominant-negative oncogenic splicing variant of ASPP2 (ASPP2 $)$ ) (Schittenhelm et al., 2019) is aberrantly expressed in human CRC. We find inhibition of therapy-induced apoptosis as well as an increase in tumor cell proliferation and migration. Our data provides the rationale for further investigations addressing the role of $A S P P 2 \kappa$ as a potentially clinically useful biomarker and therapeutic target in CRC.

\section{MATERIALS AND METHODS}

\section{Cell Lines}

Two colorectal cancer cell lines (DLD-1 p53+/+ and HCT116 p53+/+), both a gift from Prof. Vogelstein (John-HopkinsUniversity, Baltimore, MD) were maintained in McCoy's 5A
TABLE 1 | Primers used for qRT-PCR.

\begin{tabular}{ll} 
Primer name & \multicolumn{1}{c}{ Sequence $\mathbf{( 5}^{\prime} \mathbf{-} \mathbf{3}^{\prime} \mathbf{)}$} \\
\hline ASPP2 $\kappa$ forward & CTGCTGATAGTGATGGATGGAG \\
ASPP2 $\kappa$ reverse & CCCAAAGCGCATAAATGACT \\
18 s forward & ATCCCTGAAAAGTT \\
18 s reverse & CACACCCTTAATGGC
\end{tabular}

(modified) Media (Gibco) supplemented with $10 \%$ foetal bovine serum (FBS) (Sigma Aldrich), 1\% penicillin-streptomycin (Biochrom), 1\% Sodium pyruvate and 1\% Non-Essential Amino acids (100X) (Gibco). For lentiviral particle production, HEK293T cells (Thermo Fisher) were maintained in HycloneDulbecco's Minimum Essential Media (DMEM, Gibco), supplemented with $10 \% \mathrm{FBS}$ and $200 \mu \mathrm{M}$ L-glutamine. All cell lines were kept in an incubator at $37^{\circ} \mathrm{C}$ in $5 \% \mathrm{CO} 2$.

\section{Patient Tissue Analysis}

Fresh, snap-frozen tissue, collected from 15 consented patients diagnosed with primary CRC (G2-G3) was obtained from the central Biobank of the Comprehensive Cancer Centre TübingenStuttgart. The project was approved by the local ethics committee $(188 / 2018 B O 2)$. Tumor-free surrounding tissue from the same patients served as controls.

\section{RNA Extraction and Real Time-Quantitative Polymerase Chain Reaction}

For RNA preparation and qRT-PCR, RNA was extracted using the RNeasy ${ }^{\circledast}$ RNA purification kit (Qiagen). cDNA synthesis was performed using the Reverse Transcriptase Kit (Roche). To determine ASPP $2 \kappa$ expression levels, a qRT-PCR assay was established to run with qRT-PCR Roche LightCycler Technology (Roche) and Light Cycler 480 Probes Master (Roche). 18s served as housekeeping reference gene. All primer sets were purchased from Eurofins and are shown in Table 1. Measurements were performed in triplicates. Relative quantification of the target gene transcript in comparison to the reference transcript was calculated using the $\mathrm{Cp}$ method.

\section{ASPP2א Isoform-Specific Interference}

Recombinant lentiviral particles expressing a custom-made 29mer short hairpin (sh) RNA against $A S P P 2 \kappa$ were produced and transduced into the CRC cells according to the manufacturer's protocols. Empty vector (EV) strains served as controls.

Briefly, a pre-selected custom synthesized lentiviral construct was designed (pGFP-C-shLenti vector; Origene) containing a shRNA expression cassette against $A S P P 2 \kappa$, driven by an U6 promoter, a puromycin resistance marker, driven by a SV40 promoter and a tGFP, driven by a CMV promoter. HEK293T cells were lipofected (Lipofectamine 2000; Thermo Fisher) for lentiviral particle production following the manufacturer's protocol (packaging mix, Dharmacon). The lentiviral particles were used to transduce HCT116 or DLD-1 cells to induce stable hairpin expression against $A S P P 2 \kappa$. The transduction efficiency was 
evaluated determining GFP expression, while puromycin was used as a selection marker.

\section{Enforced ASPP2к Expression Model}

A lipofection approach was followed to create ASPP $2 \kappa$ overexpressing cell lines, using a custom-made HisMax vector (Eurofins) encoding the peptide sequences of the ASPP $2 \kappa$ splicing variant and a Zeocin resistance site. A HisMax LacZ vector served as an empty vector control (Schittenhelm et al., 2019).

\section{APOPTOSIS ASSAY}

Cells were seeded 1 day prior to treatment at $40-50 \%$ confluency. Oxaliplatin (Ox) was obtained from Sigma-Aldrich and dissolved in $\mathrm{H} 2 \mathrm{O}$. A dose-dilution assay was set up and cells were cultured for $48 \mathrm{~h}$ at $37^{\circ} \mathrm{C}$. Cells were then harvested and stained for AnnexinV/PI or APC/7AAD according to manufacturer's guidelines (Life Technologies) and measured on a FACS Calibur (Becton Dickinson). Experiments were performed in triplicates.

\section{Proliferation Assay}

The number of living cells per well was assessed daily using a hemacytometer after trypan blue staining. Experiments were performed in technical triplicates. Cellular doubling times were determined using the following formula:

$$
t_{d}=\frac{\ln \ln (2) * t}{x_{t}-x_{0}}
$$

$\mathrm{t}_{\mathrm{d}}=$ doubling time[h]. $\mathrm{t}=$ duration $[\mathrm{h}] . \mathrm{x}_{0}=$ cell number at timepoint $0 . \mathrm{x}_{\mathrm{t}}=$ final cell number at timepoint $\mathrm{t}$.

\section{Wound Healing Assay}

For the wound healing assay, cells were grown to $100 \%$ confluency. A $20 \mu \mathrm{l}$ pipette tip was used to create a defined scratch wound. Wound closure was monitored by light microscope (Nikon) with 10x magnification every $2 \mathrm{~h}$ for a total of $12 \mathrm{~h}$ and additionally after $24 \mathrm{~h}$. Cell migration was quantified and compared to the corresponding control cell strains using NIS Elements software (Nikon).

\section{Transwell Migration Assay}

To determine the migration capacity, a transwell migration assay was established: Cells were seeded in $1.5 \mathrm{ml} \mathrm{McCoy}$ (Gibco) pure medium into the upper transwell migration inserts with $8 \mu \mathrm{m}$ pores. $2.5 \mathrm{ml} \mathrm{20 \%}$ FBS McCoy medium were added to the bottom chamber. After allowing the cells to migrate for $16 \mathrm{~h}$, all cells from the upper insert including the top of the membrane were removed using a cotton tip. The penetrated cells in the bottom chamber, including cells attached to the bottom side of the transwell membrane, were collected, fixed with $70 \%$ ethanol, and stained with a Giemsa (Merck KGaA) solution. Experiments were performed in triplicates. For quantitative analysis, a picture was taken from five random fields using a light microscope (Nikon) at a magnification of $20 \mathrm{x}$ and the migrated cells were counted (Mohle et al., 2000).

\section{Statistical Analysis}

Statistical analyses were carried out using GraphPad Prism. Quantitative variables were analysed by Student's paired and/ or unpaired $t$-test, and 2way ANOVA as indicated. All statistical analyses were two-sided, and $p<0.05$ was considered statistically significant.

\section{RESULTS}

\section{Detection of ASPP2к in Human CRC}

To determine if $A S P P 2 \kappa$ is expressed in human CRC, an isoformspecific qRT-PCR assay was established to screen for ASPP $2 \kappa$ mRNA expression levels in snap-frozen primary tumor tissue obtained from 15 patients with newly diagnosed adenocarcinoma (G2/3). Patient characteristics are provided as Supplementary Table S1. A pool of tumor-free colon tissue $(n=14)$ was used as a control cohort.

We found that on average, $A S P P 2 \kappa$ is significantly overexpressed in CRC tumor samples compared to normal tissue $(p<0.001)$. Interestingly, expression levels varied widely, ranging from non-expressors to a $>10$-fold increase in a subset of samples (Figure 1A).

Comparison of individual paired tumor vs. adjacent microscopically tumor-free colon tissue samples $(\mathrm{n}=11)$ revealed significant upregulation of $A S P P 2 \kappa$ in approx. half of the tested samples. Importantly, expression of $A S P P 2 \kappa$ was thereby specifically linked to the tumor tissue (Figure 1B).

\section{ASPP2 $\kappa$ Is Stress-Inducible in CRC}

We reported previously that $A S P P 2 \kappa$ expression is inducible in leukemia (Schittenhelm et al., 2019) in response to different stressors including temperature, UV light or chemotherapy. To explore if $A S P P 2 \kappa$ expression is stress-inducible in CRC, we utilized CRC human cell lines DLD-1 (Dukes CRC; TP53 WT (Vives et al., 2006)) and HCT116 (poorly differentiated CRC from primary site; TP53 WT (Sullivan and Lu, 2007)). Cells were cultured at $37^{\circ} \mathrm{C}$ or room temperature (RT) overnight and mRNA levels of $A S P P 2 \kappa$ were quantified in relation to a baseline. In both cell lines, exposure to room temperature stress resulted in a statistically significant induction of $A S P P 2 \kappa$ expression compared to $37^{\circ} \mathrm{C}$ (Figure 1C).

\section{ASPP2к Expression Modulates CRC Sensitivity to Chemotherapy-Induced Apoptosis}

In order to assess whether ASPP $2 \kappa$ has an effect on induction of chemotherapy-induced apoptosis, we modified ASPP $2 \kappa$ expression in DLD-1 and HCT116 cell lines, exposed them to the DNA-intercalating agent oxaliplatin (Ox) (a clinically important therapeutic for patients with CRC) and then measured the degree of induction of apoptosis. To silence ASPP2 $\kappa$, we used an isoform-specific shRNA approach as confirmed by qRT-PCR for each cell line, showing a decrease in ASPP $2 \kappa$ expression of $27 \%$ for DLD- 1 and $21 \%$ for HCT 116 (Figure 2A). To enforce ASPP2 $\kappa$ expression, the same cell lines 

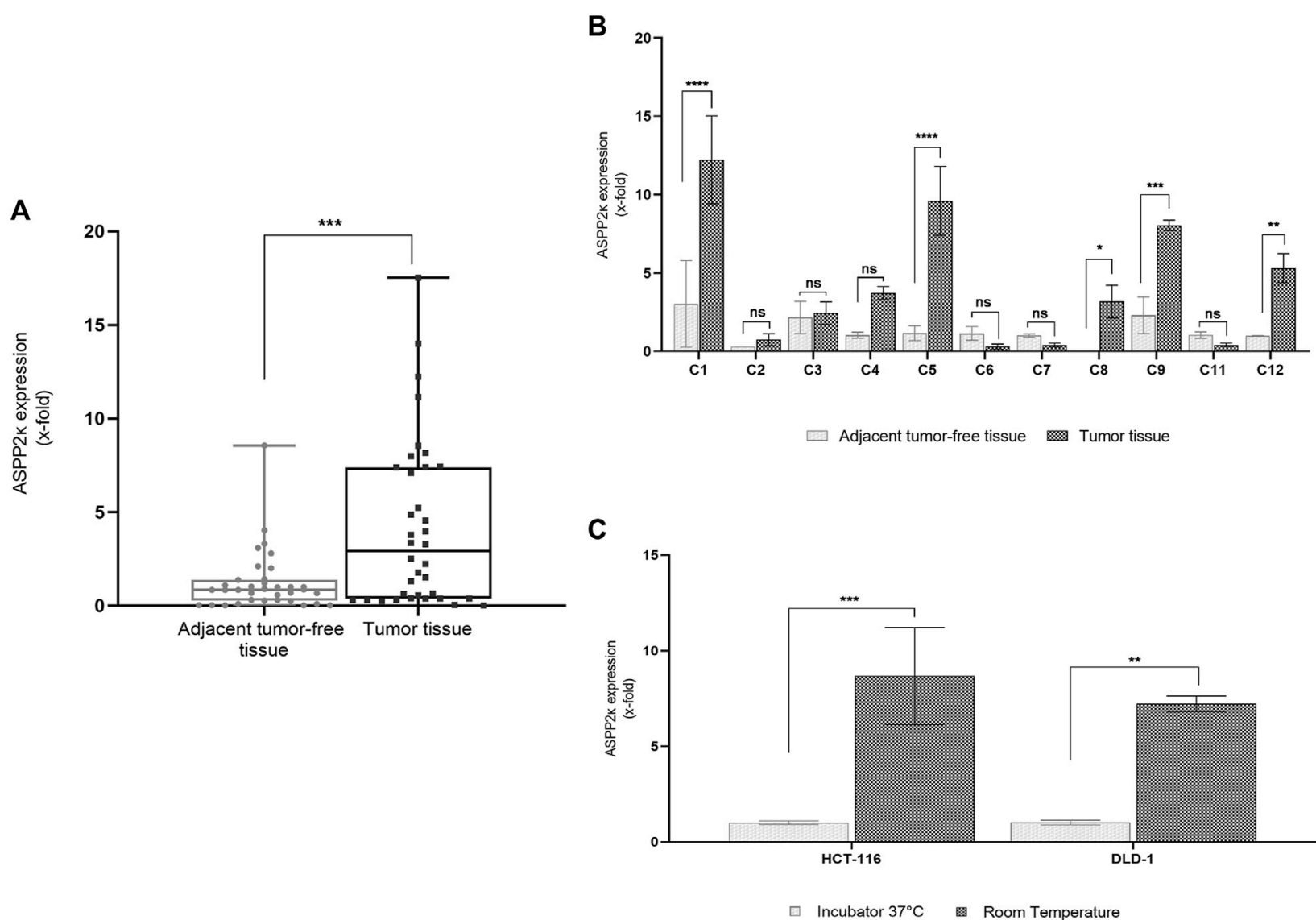

FIGURE 1 | (A) ASPP2 $\kappa$ isoform-specific qRT-PCR analysis determines relative mRNA expression levels in CRC tissue $(n=15)$ compared to a pool of adjacent tumor-free tissue colorectal tissue $(n=14)$. Analyses performed in triplicates. Statistical tests: unpaired $t$-test (B) ASPP2 $\kappa$ specific qRT-PCR assay to compare individual ASPP2 $\kappa$ expression levels in tumor tissue vs. adjacent tumor-free tissue colorectal tissue of the same patient. Statistical test: two-way ANOVA. Pair C10 was removed from analysis due to R1 resection status. (C) ASPP2 $\kappa$ specific qRT-PCR demonstrates temperature inducible expression of the splicing variant in CRC cell lines. Statistical test: two-way ANOVA. ${ }^{\star \star \star \star} p<0.0001,{ }^{\star \star \star} p<0.001,{ }^{\star \star} p<0.01,{ }^{\star} p<0.05$, ns: not statistically significant.
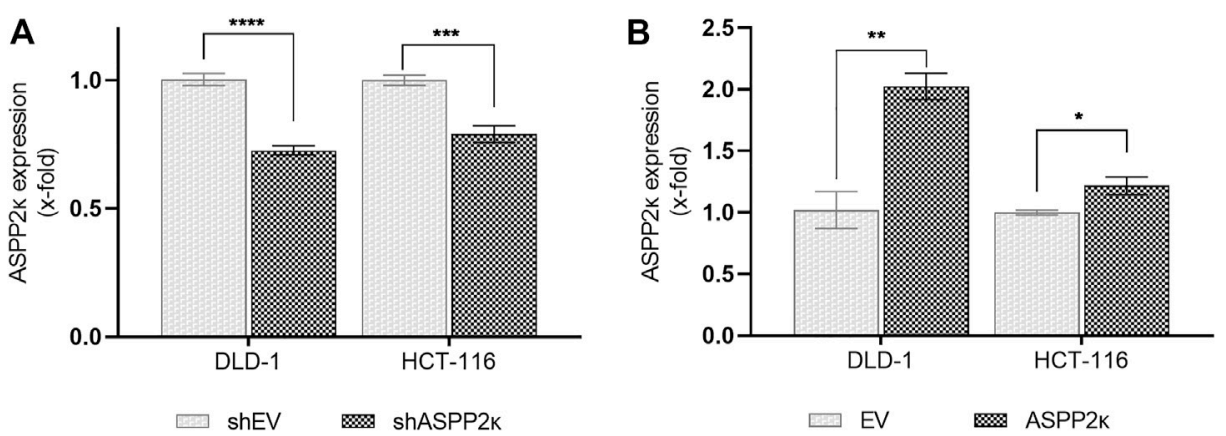

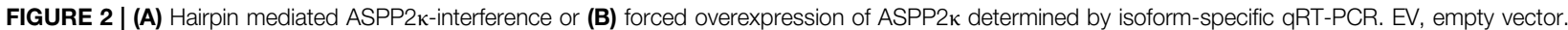
Statistical test: two-way ANOVA. ${ }^{\star \star \star \star} p<0.0001,{ }^{\star \star \star} p<0.001,{ }^{* \star} p<0.01,{ }^{\star} p<0.05$.

were transfected with a HisMax vector encoding for $A S P P 2 \kappa$ using a lipofection approach. Overexpression of $A S P P 2 \kappa$ was achieved with a 2 -fold increase in the DLD-1 model and a $20 \%$ increase of expression levels for the HCT116 cell line (Figure 2B).

Given that ASPP2 $\kappa$ promotes resistance to apoptosis in human leukemia, we reasoned that enforced expression in CRC cells would promote resistance to apoptosis. As expected, ASPP2א interferenced cell lines were more sensitive to oxaliplatininduced apoptosis compared to EV controls (Figures 3A,C). In contrast, cell lines overexpressing ASPP2 $\kappa$ were more resistant to apoptosis induction in response to oxaliplatin when compared to the EV control strains (Figures 3B,D). Interestingly, even though 

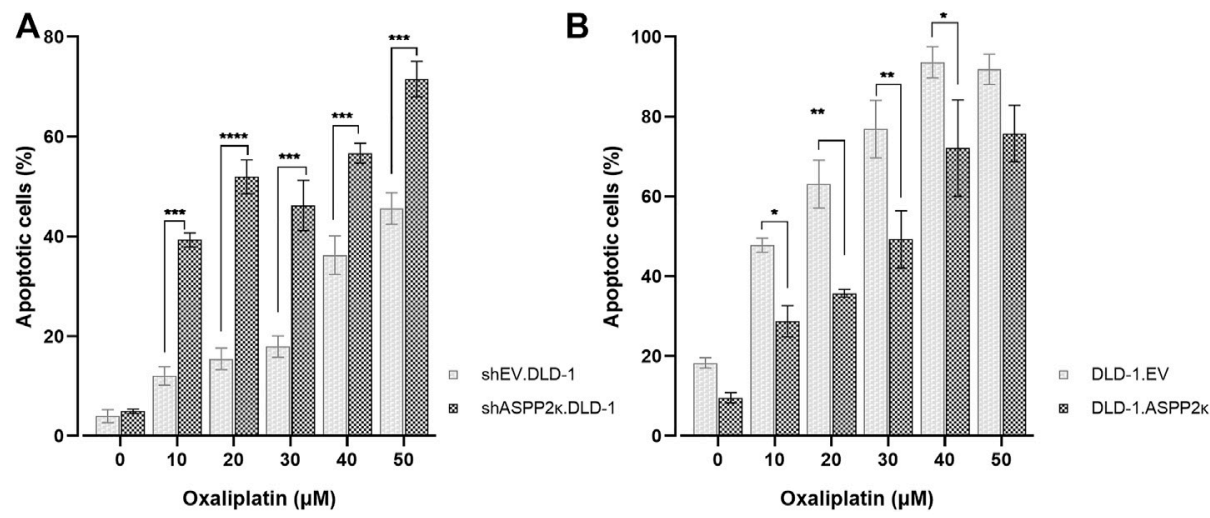

C

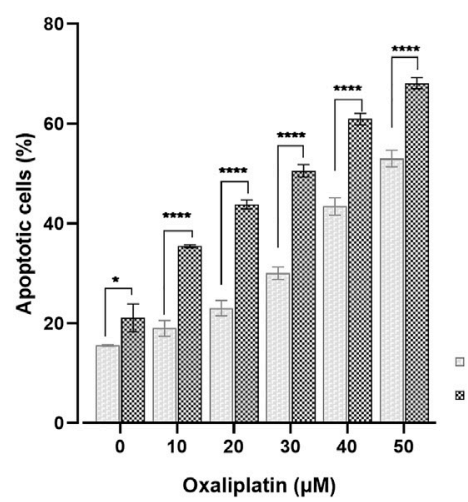

D

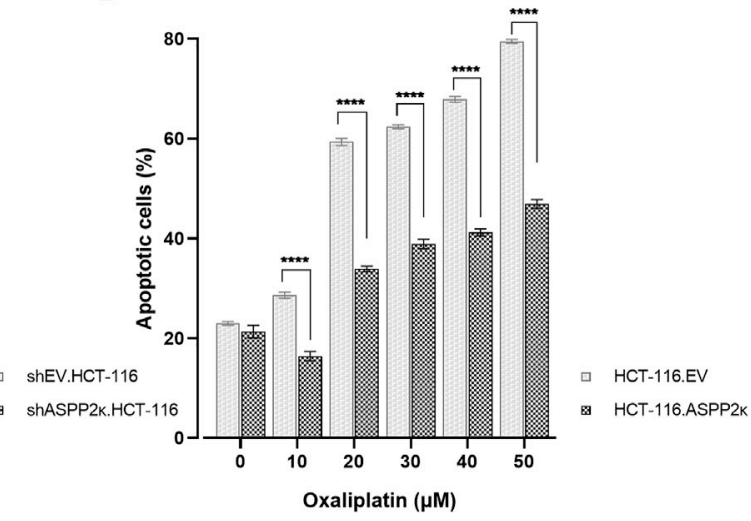

FIGURE 3 | Dose dilution experiments using (A) DLD-1 or (C) HCT116 ASPP2 $\kappa$ silenced strains (B) DLD-1 or (D) HCT116 ASPP2 $\kappa$ overexpressing strains are shown. Induction of apoptosis after exposure of cell strains to Ox for $48 \mathrm{~h}$ is assessed flow cytometrically using annexin V/7-AAD. EV, empty vector. Statistical test: twoway ANOVA. ${ }^{\star \star * \star} p<0.0001,{ }^{* \star *} p<0.001,{ }^{* \star} p<0.01,{ }^{*} p<0.05$.

the degree of $A S P P 2 \kappa$ knockdown and overexpression was modest ( $<2$-fold), yet the effect on oxaliplatin-induced apoptosis was significant. This data underscores the important dominant-negative function of the ASPP2 $\kappa$ splicing variant (Schittenhelm et al., 2019).

\section{ASPP2к Promotes Cellular Proliferation}

Since ASPP $2 \kappa$ harbors pro-tumorigenic functions (Schittenhelm et al., 2019), we wished to explore how ASPP2 $\kappa$ altered cellular proliferation. We therefore quantified cell doubling times of DLD-1 and HCT116 cell lines modified for ASPP $2 \kappa$ expression. Consistent with a pro-tumorigenic function, forced expression of ASPP $2 \kappa$ results in a significant increase in cellular proliferation rates compared to the empty vector (EV) control cells. Cell doubling times of ASPP $2 \kappa$ enforced expression DLD-1 cells was reduced by $16 \%$ from $26.4 \mathrm{~h}$ (EV) to $22.3 \mathrm{~h}$ leading to a significant increase of proliferation rates at 72 and $96 \mathrm{~h}$ (Figure 4A). A similar finding was observed for HCT116 ASPP2 $\kappa$ overexpressing cells with a reduction of cell doubling times by $14 \%$ from $19.7 \mathrm{~h}(\mathrm{EV})$ to $17 \mathrm{~h}$, which results in a statistically significant increase of proliferation rates over time (Figure 4C). In contrast, the ASPP $2 \kappa$-attenuated cell strains demonstrated an increase in cell doubling times by approximately $10 \%$ from $24.4 \mathrm{~h}(\mathrm{EV})$ to $26.9 \mathrm{~h}$ for the DLD-1 cell line, and from 19.5 to $21.2 \mathrm{~h}$ for the HCT116 strains-both leading to a significant decrease in cellular proliferation rates at 72 and $96 \mathrm{~h}$ (Figures 4B,D).

These observations are consistent with the notion that ASPP $2 \kappa$ harbors significant dominant-negative functions that promote CRC tumorigenesis.

\section{ASPP2к Enhances Cellular Migration}

Since tumor cell migration and invasion are hallmarks of cancer development (Hanahan and Weinberg, 2000), we wished to evaluate how ASPP $2 \kappa$ levels influence the migratory behaviour of CRC cells. Using an established transwell migration assay as previously described (Mohle et al., 2000), we found that forced expression of ASPP $2 \kappa$ resulted in an enhancement of cellular migration with approximately 36 and $58 \%$ higher migration rates for DLD-1ASPP $2 \kappa$ and HCT116-ASPP $2 \kappa$ cell lines when compared to the corresponding EV control cell lines (Figures 5A,C).

Vice versa, ASPP $2 \kappa$-interference resulted in attenuated cellular migration in both CRC cell lines when compared to the respective EV controls. Specifically, DLD-1 cells showed 37\% less cellular migration capacity in the transwell migration assay compared to their isogenic EV controls (Figure 5B). In HCT116 cells, ASPP2 $\kappa$ interference led to a $30 \%$ reduction of migrating cells compared to 


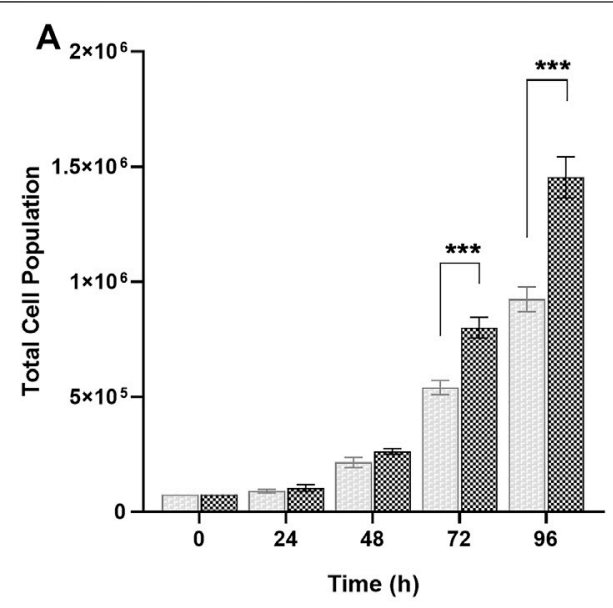

冈 DLD-1.ASPP2K $\square$ DLD-1.EV

C

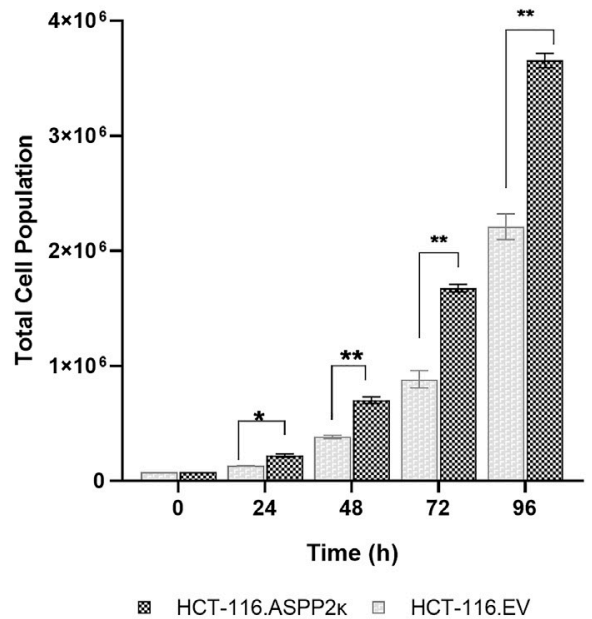

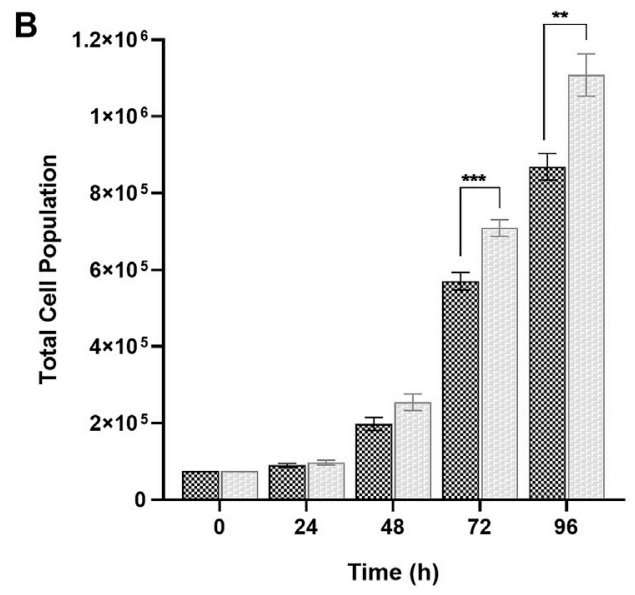

\$ ShASPP2K.DLD-1 1 shEV.DLD-1

D

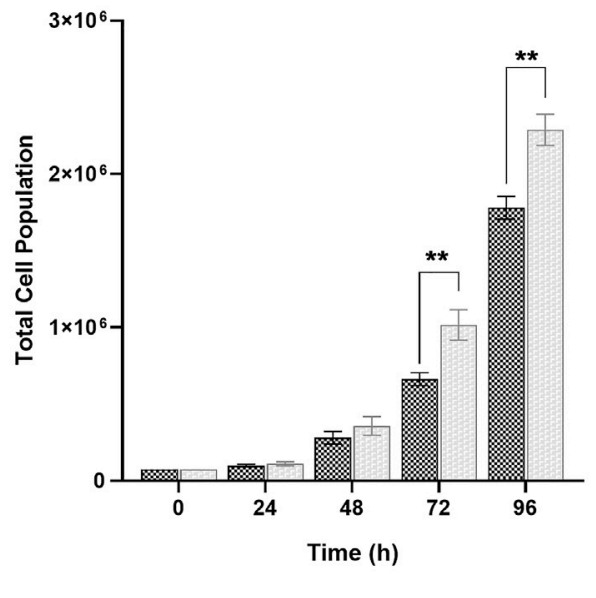

圆 shASPP2к.HCT-116 घ ShEV.HCT-116

FIGURE 4 | Cell doubling times of CRC cell lines in dependence of ASPP2 $\kappa$ : (A) DLD-1 or (C) HCT116 strains overexpressing the ASPP2 $\kappa$ isoform or (B) DLD-1 or (D) HCT116 ASPP2 $\kappa$ silenced strains are shown. EV, empty vector. Statistical test: two-way ANOVA. ${ }^{\star \star \star} p<0.001,{ }^{\star \star} p<0.01,{ }^{\star} p<0.05$.

the EV control (Figure 5D). To further confirm our findings that ASPP2 $\kappa$ enhanced CRC migration, we used a wound healing assay for the DLD-1 cell line (Figure 6). As expected, DLD-1 ASPP2 $\kappa$ overexpressing cells demonstrated enhanced cellular motility-resulting in an increased wound closure rate when compared to the DLD-1.EV control strains ( 3.3\%/h vs $2.7 \%$ / h), (Figures 6A,C). In contrast, ASPP $2 \kappa$-interference resulted in decreased cellular motility rates, which led to a delayed wound closure time of $37.5 \mathrm{~h}$ in comparison to the corresponding EV control strain wound closure time of $31 \mathrm{~h}$ (Figures 6B,D).

\section{DISCUSSION}

Although recent advances in the understanding, detection and treatment of CRC have improved outcomes for patients, CRC is still a major source of morbidity and mortality worldwide (Bray et al., 2018).

While early tumor stages can be treated curatively with surgery and chemotherapy, distant metastatic disease remains incurable except in selected circumstances (Chen et al., 2021). Therefore, understanding the cellular and molecular complexity of the disease remains crucial in order to unveil the molecular basis of CRC-tumorigenesis and to design new and more effective therapies (Ganesh et al., 2019) (Dienstmann et al., 2017).

Recent advances have shown the importance of identifying prognostic and predictive biomarkers in CRC as evidenced by several molecularly stratified treatment options. For example, the importance of microsatellite instability and TMB is evident in the use of immune-checkpoint inhibitors for specific CRC patient cohorts. Likewise, mutational status of RAS, BRAF, and ERBB2, as well as tumor-sidedness, are clinically relevant to guide therapy options (Sveen et al., 2020). 

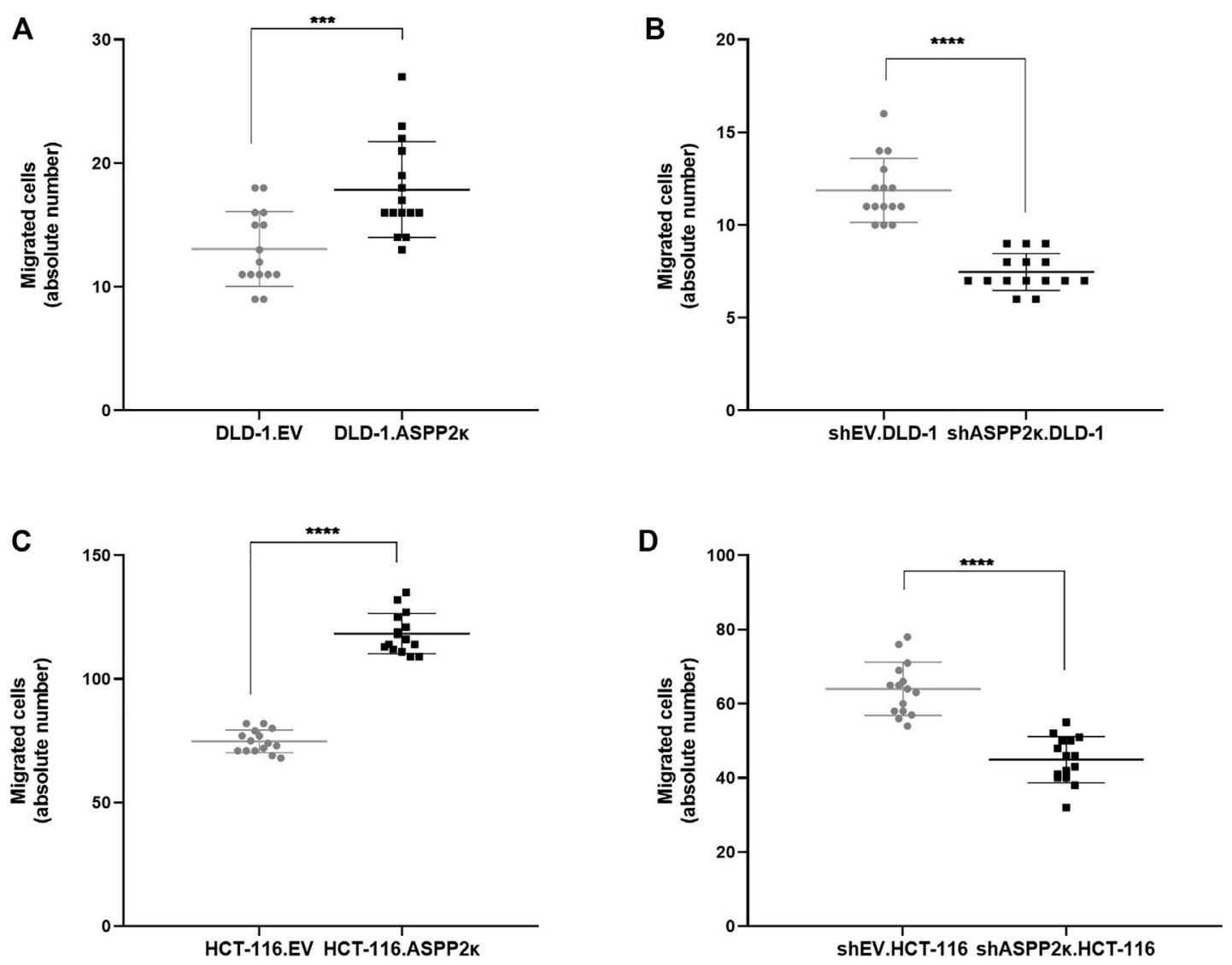

FIGURE 5 | (A-D) Quantitative analysis of migrating cells in a transwell migration assay are shown for DLD-1.ASPP2 $\kappa$ (A) and HCT-116.ASPP2 $\kappa$ (C) cells, resp. the ASPP2 $\kappa$-interferenced corresponding cell strains for DLD-1 (B) and HCT116 (D). Statistical tests: unpaired $t$-test. ${ }^{\star \star \star \star} p<0.0001,{ }^{\star \star \star} p<0.001,{ }^{\star \star} p<0.01,{ }^{\star} p<0.05$.

ASPP2 is a tumor suppressor that enhances apoptosis and promotes tumorigenesis via p53-dependent (Samuels-Lev et al., 2001) and p53-independent pathways (Kampa et al., 2009b; Wang et al., 2013). A role of dysfunctional ASPP2 in human neoplasms has been suggested by the observation that ASPP2 is downregulated in several tumor types, including human acute leukemia (Schittenhelm et al., 2013), choriocarcinoma (Mak et al., 2013), pancreatic cancer (Song et al., 2015) and diffuse large B-cell lymphoma (Lossos et al., 2002). In these studies, attenuated ASPP2 expression levels were associated with metastasis and poor clinical outcome (Lossos et al., 2002; Mak et al., 2013; Schittenhelm et al., 2013; Song et al., 2015). However, the precise mechanisms of ASPP2 regulation and function in these different contexts remain to be clarified. Further, different ASPP2 isoforms have been described, which have a profound functional impact on tumorigenesis and therapy resistance (Van Hook et al., 2017) (Schittenhelm et al., 2019).

In this context, we recently identified an exon-skipping splicing variant of ASPP2, named ASPP $2 \kappa$, a C-terminally truncated isoform lacking the p53 binding sites, and defective in promoting stress-induced dependent apoptosis (Schittenhelm et al., 2019). In this report, we measure ASPP $2 \kappa$ expression in colorectal tumors obtained from patients and importantly find that ASPP $2 \kappa$ is overexpressed in tumor tissue compared to adjacent tumor-free tissue (Figure 1). In this small dataset, higher $A S P P 2 \kappa$ expression levels were associated with clinical risk features-as each of the high expressors displayed at least one risk factor (such as tumor location, higher tumor infiltration stages, nodular involvement, distant metastasis or adverse mutations (Supplementary Table S1). However, the restricted number of patients clearly prevents a more profound analysis-and this topic has to be addressed in future studies using well-defined patient cohorts.

Intriguingly, we also found significant variation in ASPP $2 \kappa$ expression levels (Figures 1A,B). This suggests that additional pathways may be important in modulating ASPP $2 \kappa$ function. Consistent with a functional role in colorectal cancer therapeutics, we found that ASPP $2 \kappa$ expression inhibited oxaliplatin-induced apoptosis (Figure 3). Given that ASPP $2 \kappa$ is missing the C-terminal domain responsible for binding $\mathrm{p} 53$, this suggests its function may be involved in the p53-mediated pathway (Kampa et al., 2009b). Indeed, both CRC cell lines used harbored a TP53 WT background. Still, given the molecular heterogeneity in CRC (Sveen et al., 2020), including RAS/ BRAF mutations, and that ASPP2 has other binding partners such as RAS (Wang et al., 2013), the precise mechanisms of ASPP $2 \kappa$ function remain to be elucidated. Nevertheless, our finding that ASPP $2 \kappa$ expression has functional consequences 


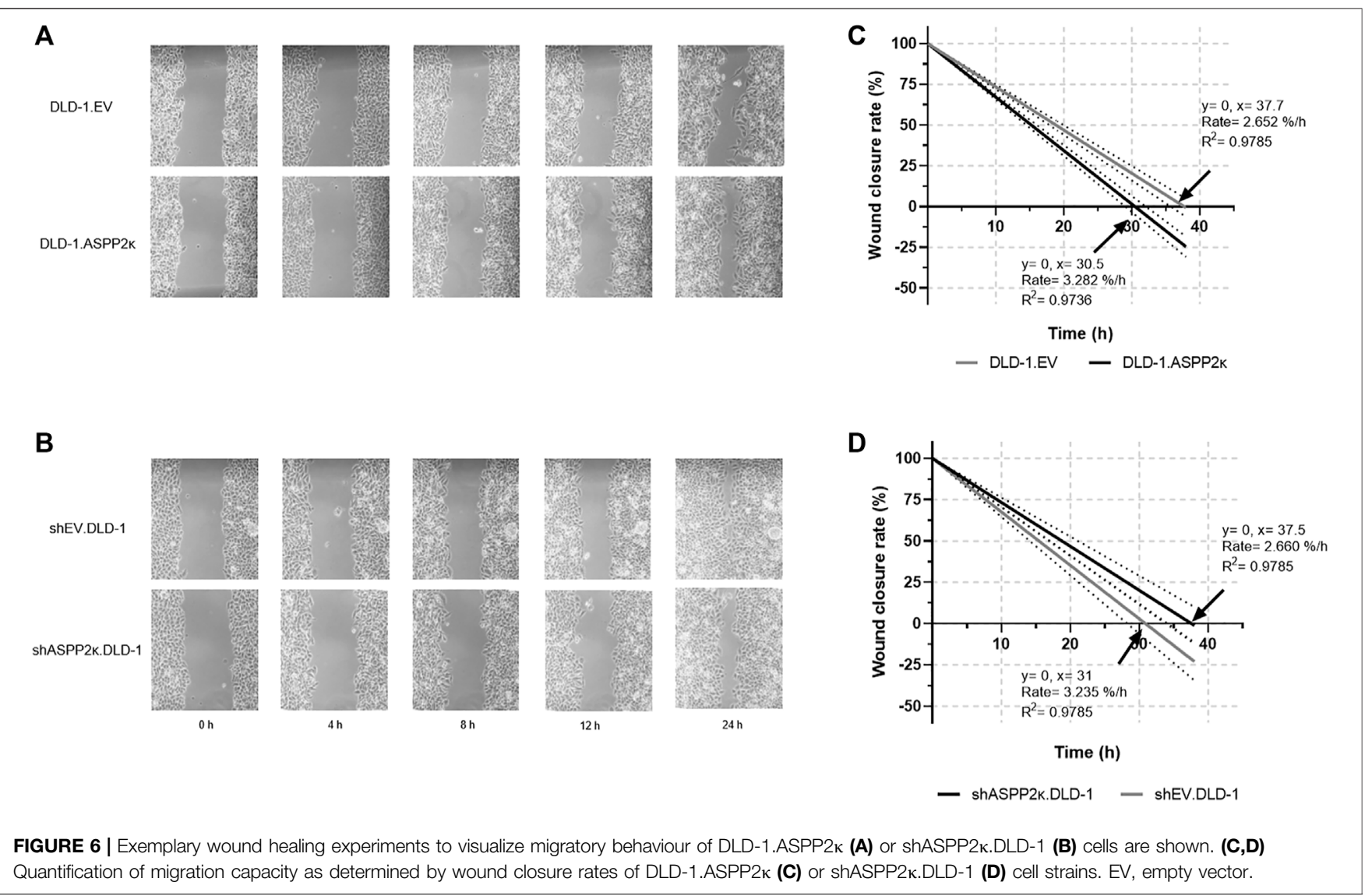

that mediate CRC tumor response to the key chemotherapy agent oxaliplatin opens important new avenues for investigation.

Previous studies have suggested that ASPP2 has other functions in addition to modulating apoptosis (Helps et al., 1995; Naumovski and Cleary, 1996; Chen et al., 2003; Meek et al., 2004; Kobayashi et al., 2005; Takahashi et al., 2005; Hakuno et al., 2007; Kampa et al., 2009a; Kampa et al., 2009b; UhlmannSchiffler et al., 2009; Wang et al., 2013). Using ASPP2א enforced expression and ASPP $2 \kappa$ silencing in CRC cell lines, we found that ASPP $2 \kappa$ promotes tumor cell proliferation and migration. These tantalizing findings argue that ASPP $2 \kappa$ has additional functions beyond apoptosis modulation and suggests an important role in CRC tumor development. Given the stepwise development of colorectal tumors from polyp to invasive cancer (Fearon and Vogelstein, 1990), it will be interesting to interrogate ASPP $2 \kappa$ expression in pre-cancerous or early-stage lesions, which may reveal important mechanism in CRC oncogenesis that may have clinical therapeutic or early detection implications.

A potential role for the ASPP family in CRC has been suggested in previous studies (Yin et al., 2018). However, these studies did not distinguish between different full-length ASPP2 and other isoforms (such as deltaN-ASPP2 (Van Hook et al., 2017) or ASPP2א (Schittenhelm et al., 2019)). This would be important because these different (and potentially functional counteracting) isoforms will make it difficult to establish robust clinical correlations. Our data strongly suggests that future studies will need to take the presence of different isoforms into account to understand the larger complex role of ASPP2 in colorectal cancer.

In summary, our findings demonstrate that ASPP $2 \kappa$ is expressed in human CRC tumors and that this may play a role in resistance to oxaliplatin-based chemotherapy. Moreover, our data also demonstrates that ASPP $2 \kappa$ may play a role in colorectal tumor development as evidenced by its ability to promote tumor cell proliferation and migration.

We provide important information that sets the stage for a larger scale investigation into the role of ASPP $2 \kappa$ as a prognostic and predictive biomarker that may play a role in making a positive impact for patients with colorectal cancer.

\section{DATA AVAILABILITY STATEMENT}

The original contributions presented in the study are included in the article/Supplementary Material, further inquiries can be directed to the corresponding author.

\section{ETHICS STATEMENT}

The studies involving human participants were reviewed and approved by the Comprehensive Cancer Centre Tübingen- 
Stuttgart after approval of the project by the local ethics committee (188/2018BO2). The patients/participants provided their written informed consent to participate in this study.

\section{AUTHOR CONTRIBUTIONS}

IR designed the research study, performed the functional models, analyzed the data, and wrote the paper. VT designed the research study, performed the functional models, analyzed the data, and wrote the paper. MO provided patient information, FF provided the patient tissue, $\mathrm{CL}$ analyzed data and wrote the paper. MS and KK-S designed the research study, analyzed clinical and experimental data, and wrote the paper.

\section{REFERENCES}

Ahn, J., Byeon, I.-J. L., Byeon, C.-H., and Gronenborn, A. M. (2009). Insight into the Structural Basis of Pro- and Antiapoptotic P53 Modulation by ASPP Proteins. J. Biol. Chem. 284, 13812-13822. doi:10.1074/jbc.m808821200

Bergamaschi, D., Samuels, Y., Jin, B., Duraisingham, S., Crook, T., and Lu, X. (2004). ASPP1 and ASPP2: Common Activators of P53 Family Members. Mol. Cel Biol 24 (3), 1341-1350. doi:10.1128/mcb.24.3.1341-1350.2004

Bergamaschi, D., Samuels, Y., Sullivan, A., Zvelebil, M., Breyssens, H., Bisso, A., et al. (2006). iASPP Preferentially Binds P53 Proline-Rich Region and Modulates Apoptotic Function of Codon 72-polymorphic P53. Nat. Genet. 38 (10), 1133-1141. doi:10.1038/ng1879

Bray, F., Ferlay, J., Soerjomataram, I., Siegel, R. L., Torre, L. A., and Jemal, A. (2018). Global Cancer Statistics 2018: GLOBOCAN Estimates of Incidence and Mortality Worldwide for 36 Cancers in 185 Countries. CA: A Cancer J. Clinicians 68 (6), 394-424. doi:10.3322/caac.21492

Chen, E. Y., Mayo, S. C., Sutton, T., Kearney, M. R., Kardosh, A., Vaccaro, G. M., et al. (2021). Effect of Time to Surgery of Colorectal Liver Metastases on Survival. J. Gastrointest. Canc 52 (1), 169-176. doi:10.1007/s12029-02000372-5

Chen, Y., Liu, W., Naumovski, L., and Neve, R. L. (2003). ASPP2 Inhibits APPBP1-Mediated NEDD8 Conjugation to Cullin-1 and Decreases APP-BP1Induced Cell Proliferation and Neuronal Apoptosis. J. Neurochem. 85 (3), 801-809. doi:10.1046/j.1471-4159.2003.01727.x

Dienstmann, R., Vermeulen, L., Guinney, J., Kopetz, S., Tejpar, S., and Tabernero, J. (2017). Consensus Molecular Subtypes and the Evolution of Precision Medicine in Colorectal Cancer. Nat. Rev. Cancer. 17 (2), 79-92. doi:10.1038/nrc.2016.126

Fearon, E. R., and Vogelstein, B. (1990). A Genetic Model for Colorectal Tumorigenesis. cell 61 (5), 759-767. doi:10.1016/0092-8674(90)90186-i

Ganesh, K., Stadler, Z. K., Cercek, A., Mendelsohn, R. B., Shia, J., Segal, N. H., et al. (2019). Immunotherapy in Colorectal Cancer: Rationale, Challenges and Potential. Nat. Rev. Gastroenterol. Hepatol. 16 (6), 361-375. doi:10.1038/ s41575-019-0126-x

Gorina, S., and Pavletich, N. P. (1996). Structure of the P53 Tumor Suppressor Bound to the Ankyrin and SH3 Domains of 53BP2. Science. 274 (5289), 1001-1005. doi:10.1126/science.274.5289.1001

Hakuno, F., Kurihara, S., Watson, R. T., Pessin, J. E., and Takahashi, S.-I. (2007). 53BP2S, Interacting with Insulin Receptor Substrates, Modulates Insulin Signaling. J. Biol. Chem. 282 (52), 37747-37758. doi:10.1074/jbc.m702472200

Hanahan, D., and Weinberg, R. A. (2000). The Hallmarks of Cancer. Cell 100, $57-70$.

Helps, N. R., Barker, H. M., Elledge, S. J., and Cohen, P. T. (1995). Protein Phosphatase 1 Interacts with p53BP2, a Protein Which Binds to the Tumour Suppressor P53. FEBS Lett. 377 (3), 295-300. doi:10.1016/0014-5793(95) $01347-4$

\section{FUNDING}

Unrestricted grant support by the Brigitte Schlieben-Lange Program as well as the Margarete von Wrangell Program of the Ministry of Science, Research and the Arts, Baden-Württemberg, Germany (KK-S) and Athene Program of the excellence initiative of the University of Tübingen (KK-S), DGVS doctoral thesis scholarship (IR). This study was supported by the central biobank of the comprehensive cancer center (CCC) Tübingen-Stuttgart.

\section{SUPPLEMENTARY MATERIAL}

The Supplementary Material for this article can be found online at: https:/www.frontiersin.org/articles/10.3389/fmolb.2021.727203/ full\#supplementary-material

Iwabuchi, K., Bartel, P. L., Li, B., Marraccino, R., and Fields, S. (1994). Two Cellular Proteins that Bind to Wild-type but Not Mutant P53. Proc. Natl. Acad. Sci. 91 (13), 6098-6102. doi:10.1073/pnas.91.13.6098

Iwabuchi, K., Li, B., Massa, H. F., Trask, B. J., Date, T., and Fields, S. (1998). Stimulation of P53-Mediated Transcriptional Activation by the P53-Binding Proteins, 53BP1 and 53BP2. J. Biol. Chem. 273 (40), 26061-26068. doi:10.1074/ jbc.273.40.26061

Kampa, K. M., Acoba, J. D., Chen, D., Gay, J., Lee, H., Beemer, K., et al. (2009). Apoptosis-stimulating Protein of P53 (ASPP2) Heterozygous Mice Are TumorProne and Have Attenuated Cellular Damage-Response Thresholds. Proc. Natl. Acad. Sci. 106 (11), 4390-4395. doi:10.1073/pnas.0809080106

Kampa, K. M., Bonin, M., and Lopez, C. D. (2009). New Insights into the Expanding Complexity of the Tumor Suppressor ASPP2. Cell Cycle 8 (18), 2871-2876. doi:10.4161/cc.8.18.9474

Kobayashi, S., Kajino, S., Takahashi, N., Kanazawa, S., Imai, K., Hibi, Y., et al. (2005). 53BP2 Induces Apoptosis through the Mitochondrial Death Pathway. Genes Cells 10 (3), 253-260. doi:10.1111/j.1365-2443.2005.00835.x

Lossos, I. S., Natkunam, Y., Levy, R., and Lopez, C. D. (2002). Apoptosis Stimulating Protein of P53 (ASPP2) Expression Differs in Diffuse Large B-Cell and Follicular center Lymphoma: Correlation with Clinical Outcome. Leuk. Lymphoma 43 (12), 2309-2317. doi:10.1080/ 1042819021000040017

Mak, V. C. Y., Lee, L., Siu, M. K. Y., Wong, O. G. W., Lu, X., Ngan, H. Y. S., et al. (2013). Downregulation of ASPP2 in Choriocarcinoma Contributes to Increased Migratory Potential through Src Signaling Pathway Activation. Carcinogenesis 34 (9), 2170-2177. doi:10.1093/carcin/bgt161

Meek, S. E. M., Lane, W. S., and Piwnica-Worms, H. (2004). Comprehensive Proteomic Analysis of Interphase and Mitotic 14-3-3-binding Proteins. J. Biol. Chem. 279 (31), 32046-32054. doi:10.1074/jbc.m403044200

Mohle, R., Schittenhelm, M., and Failenschmid, C. (2000). Functional Response of Leukemic Blasts to SDF-1 Correlates with Preferential Expression of the Chemokine Receptor CXCR4 in Acute Myelomonocytic and Lymphoblasdtic Leukemia. Br. J. Haematol. 110 (7), 563-572. doi:10.1046/ j.1365-2141.2000.02157.x

Naumovski, L., and Cleary, M. L. (1996). The P53-Binding Protein 53BP2 Also Interacts with $\mathrm{Bc} 12$ and Impedes Cell Cycle Progression at G2/M. Mol. Cel Biol 16 (7), 3884-3892. doi:10.1128/mcb.16.7.3884

Samuels-Lev, Y., O’Connor, D. J., Bergamaschi, D., Trigiante, G., Hsieh, J.-K., Zhong, S., et al. (2001). ASPP Proteins Specifically Stimulate the Apoptotic Function of P53. Mol. Cel. 8 (4), 781-794. doi:10.1016/s1097-2765(01) 00367-7

Schittenhelm, M. M., Illing, B., Ahmut, F., Rasp, K. H., Blumenstock, G., Döhner, K., et al. (2013). Attenuated Expression of Apoptosis Stimulating Protein of P53-2 (ASPP2) in Human Acute Leukemia Is Associated with Therapy Failure. PLoS One 8 (11), e80193. doi:10.1371/journal.pone.0080193

Schittenhelm, M. M., Walter, B., Tsintari, V., Federmann, B., Bajrami Saipi, M., Akmut, F., et al. (2019). Alternative Splicing of the Tumor Suppressor 
ASPP2 Results in a Stress-Inducible, Oncogenic Isoform Prevalent in Acute Leukemia. EBioMedicine 42, 340-351. doi:10.1016/ j.ebiom.2019.03.028

Song, B., Bian, Q., Zhang, Y.-J., Shao, C.-H., Li, G., Liu, A.-A., et al. (2015). Downregulation of ASPP2 in Pancreatic Cancer Cells Contributes to Increased Resistance to Gemcitabine through Autophagy Activation. Mol. Cancer. 14 (1), 177. doi:10.1186/s12943-015-0447-5

Sullivan, A., and Lu, X. (2007). ASPP: a New Family of Oncogenes and Tumour Suppressor Genes. Br. J. Cancer. 96 (2), 196-200. doi:10.1038/ sj.bjc. 6603525

Sveen, A., Kopetz, S., and Lothe, R. A. (2020). Biomarker-guided Therapy for Colorectal Cancer: Strength in Complexity. Nat. Rev. Clin. Oncol. 17 (1), 11-32. doi:10.1038/s41571-019-0241-1

Takahashi, N., Kobayashi, S., Jiang, X., Kitagori, K., Imai, K., Hibi, Y., et al. (2004). Expression of 53BP2 and ASPP2 Proteins from TP53BP2 Gene by Alternative Splicing. Biochem. Biophysical Res. Commun. 315 (2), 434-438. doi:10.1016/ j.bbrc.2004.01.079

Takahashi, N., Kobayashi, S., Kajino, S., Imai, K., Tomoda, K., Shimizu, S., et al. (2005). Inhibition of the 53BP2S-Mediated Apoptosis by Nuclear Factor kappaB and Bcl-2 Family Proteins. Genes Cells 10 (8), 803-811. doi:10.1111/ j.1365-2443.2005.00878.x

Trigiante, G., and Lu, X. (2006). ASPPs and Cancer. Nat. Rev. Cancer 6 (3), 217-226. doi:10.1038/nrc1818

Uhlmann-Schiffler, H., Kiermayer, S., and Stahl, H. (2009). The DEAD Box Protein Ddx42p Modulates the Function of ASPP2, a Stimulator of Apoptosis. Oncogene 28 (20), 2065-2073. doi:10.1038/onc.2009.75

Van Hook, K., Wang, Z., Chen, D., Nold, C., Zhu, Z., Anur, P., et al. (2017). $\Delta$ NASPP2, a Novel Isoform of the ASPP2 Tumor Suppressor, Promotes Cellular Survival. Biochem. biophysical Res. Commun. 482 (4), 1271-1277. doi:10.1016/ j.bbrc.2016.12.027

Vives, V., Slee, E. A., and Lu, X. (2006). ASPP2: a Gene that Controls Life and Death In Vivo. Cell Cycle 5 (19), 2187-2190. doi:10.4161/cc.5.19.3266
Vogelstein, B., Papadopoulos, N., Velculescu, V. E., Zhou, S., Diaz, L. A., and Kinzler, K. W. (2013). Cancer Genome Landscapes. Science 339 (6127), 1546-1558. doi:10.1126/science.1235122

Wang, Z., Liu, Y., Takahashi, M., Van Hook, K., Kampa-Schittenhelm, K. M., Sheppard, B. C., et al. (2013). N Terminus of ASPP2 Binds to Ras and Enhances Ras/Raf/MEK/ERK Activation to Promote Oncogene-Induced Senescence. Proc. Natl. Acad. Sci. 110 (1), 312-317. doi:10.1073/ pnas. 1201514110

Yin, L., Lin, Y., Wang, X., Su, Y., Hu, H., Li, C., et al. (2018). The Family of Apoptosis-Stimulating Proteins of P53 Is Dysregulated in Colorectal Cancer Patients. Oncol. Lett. 15 (5), 6409-6417. doi:10.3892/ol.2018.8151

Conflict of Interest: MS and KK-S hold patents U.S. 13/753,354, PCT/EP 2011/ 063283, and GER 102010033575.4-41.

The remaining authors declare that the research was conducted in the absence of any commercial or financial relationships that could be construed as a potential conflict of interest.

Publisher's Note: All claims expressed in this article are solely those of the authors and do not necessarily represent those of their affiliated organizations, or those of the publisher, the editors and the reviewers. Any product that may be evaluated in this article, or claim that may be made by its manufacturer, is not guaranteed or endorsed by the publisher.

Copyright (c) 2021 Rieger, Tsintari, Overkamp, Fend, Lopez, Schittenhelm and Kampa-Schittenhelm. This is an open-access article distributed under the terms of the Creative Commons Attribution License (CC BY). The use, distribution or reproduction in other forums is permitted, provided the original author(s) and the copyright owner(s) are credited and that the original publication in this journal is cited, in accordance with accepted academic practice. No use, distribution or reproduction is permitted which does not comply with these terms. 


\section{GLOSSARY}

18s 18 S ribosomal RNA

7-AAD 7-Aminoactinomycin

ANOVA Analysis of Variance

APC Allophycocyanin

ASPP1 Apoptosis Stimulating Protein of p53 protein 1

ASPP2 Apoptosis Stimulating Protein of $\mathrm{p} 53$ protein 2

Bbp Bcl2-binding protein

cDNA complementary DNA

CMV Cytomegalovirus

Cp Crossing point

CRC Colorectal carcinoma

DMEM Dulbecco's Modified Eagle's Medium

EV Empty Vector

FACS Fluorescence Activated Cell Sorting

FBS Fetal Bovine Serum

GFP Green Fluorescent Protein
iASPP Inhibitor of Apoptosis Stimulating Protein of p53

IC50 Half Maximal Inhibitory Concentration

MEF Mouse Embryonic Fibroblast

mRNA messenger ribonucleic acid

Ox Oxaliplatin

PBS Phosphate Buffer Saline

PI Propidium Iodide

qRT-PCR quantitative reverse transcription polymerase chain reaction

RT Room Temperature

SH3 SRC Homology 3

shRNA short hairpin ribonucleic acid

siRNA silencing ribonucleic acid

SV40 Simian virus 40

TP53 Tumor Protein 53

TP53BP2 Tumor Protein P53 Binding Protein 2

WHO World Health Organization

WT Wild Type 